\title{
Genomics education for medical professionals - the current UK landscape 䇺
}

\author{
Authors: Ingrid Slade, ${ }^{A, *}$ Deepak N Subramanian ${ }^{B, *}$ and Hilary Burton ${ }^{C}$
}

Genomics education in the UK is at an early stage of development, and its pace of evolution has lagged behind that of the genomics research upon which it is based. As a result, knowledge of genomics and its applications remains limited among non-specialist clinicians. In this review article, we describe the complex landscape for genomics education within the UK, and highlight the large number and variety of organisations that can influence, direct and provide genomics training to medical professionals. Postgraduate genomics education is being shaped by the work of the Health Education England (HEE) Genomics Education Programme, working in conjunction with the Joint Committee on Genomics in Medicine. The success of their work will be greatly enhanced by the full cooperation and engagement of the many groups, societies and organisations involved with medical education and training (such as the royal colleges). Without this cooperation, there is a risk of poor coordination and unnecessary duplication of work. Leadership from an organisation such as the HEE Genomics Education Programme will have a key role in guiding the formulation and delivery of genomics education policy by various stakeholders among the different disciplines in medicine.

KEYWORDS: Genomics, genetics, medical education, postgraduate training, continuing professional development

\section{Introduction}

The first human genome sequence was completed in 2003, marking the beginning of a period of rapid development in the understanding of the role of genomic variation in human disease. Advances in the technologies underpinning genetic research have revealed the astonishing complexity of human biology and, although there is much investigation still to be done, notable new interventions are already applicable in the clinical setting. For example, in 1990 the genetic and molecular

Authors: A research associate, Ethox Centre, Nuffield Department of Population Health, University of Oxford, Oxford, UK; specialist registrar, Oxford University Hospitals Trust, Oxford, UK; director, Centre for Personalised Medicine, University of Oxford, Oxford, UK; ${ }^{B}$ research associate, PHG Foundation, Cambridge, UK; ' director, PHG Foundation, Cambridge, UK; *joint first authors basis was understood for fewer than $2 \%$ of the estimated 7,000 suspected Mendelian conditions; in 2013, approximately 50\% of Mendelian conditions had a known molecular basis, and it is predicted that most of the remaining disease-causing genes will be identified by $2020 .^{1,2}$ Genomic advances are also aiding the understanding of the nature of cancer, the variability of individual responses to drugs (pharmacogenomics) and the genetic determinants and mechanisms underlying common complex conditions, such as heart disease and diabetes. ${ }^{3-6}$ Genomic knowledge and approaches have the potential to aid the delivery of ever more personalised medical care and are increasingly relevant to all healthcare practitioners. Education of physicians will be instrumental in the workforce development required for the delivery of genomic medicine. ${ }^{3,7,8}$ It is increasingly acknowledged that clinical genetics services are not able to meet the current and growing demand for genetic testing, and, although it is important that clinical genetics departments continue with their current and constantly updated specialist role, a widespread integration of genetics into mainstream specialties is broadly agreed as the optimum way for health services to evolve. ${ }^{9-11}$ The appropriate and effective use of genomic information in the care of patients will require clinicians to be sufficiently versed and confident in the use of genomics and to understand when genomics should be applied, be able to communicate the benefits and limitations to their patients and, for inherited disease, recognise when specialist genetic review would be appropriate. However, clinical genetics has evolved historically as a separate clinical specialty with its own dedicated training programme. ${ }^{12}$ Therefore, although some specialties, such as cardiology and medical oncology, have incorporated genetic competencies into sections of their training curriculums, many physicians remain hesitant in the interpretation of traditional genetic tests, much less the complex genomic information obtained from new sequencing technology. ${ }^{12-14}$ There are limited examples of genomics educational success but numerous publications document that the majority of physicians are interested in genomics but lack confidence with the topic. ${ }^{14-16}$

In the UK, the 2003 genetics white paper Our inheritance, our future marked the start of an ambition for healthcare services to use advances in genomics technology and knowledge for the benefit of patients. ${ }^{17}$ To deliver on this vision, the white paper included a commitment to the education and training of healthcare staff. More recently, the 2009 House of Lords' genomic medicine report highlighted that the clinical 
Fig 1. The structure of medical training in the UK. JRCPTB = Joint Royal Colleges of Physicians Training Board.

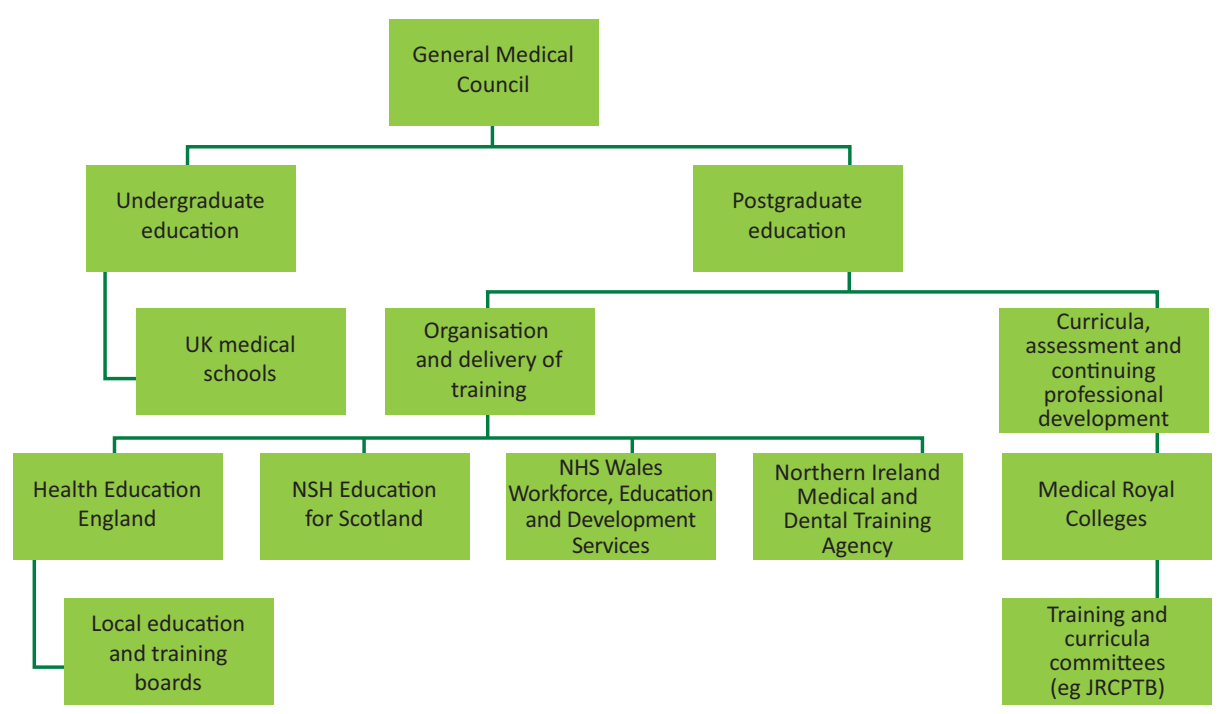

applications of genomics had not been incorporated at any scale into service provision, noting workforce education as one of the challenges in integrating these advances within the healthcare service. This report, and subsequent others, have included calls for organisations involved in the specialist training of physicians, such as the royal colleges, to recognise the importance of genomics within the practice of all clinical subspecialties and to develop appropriate training programmes. $3,8,18-20$

In 2012, the UK government launched a project to carry out the whole-genome sequencing of 100,000 patients within the NHS. This ambitious project is built upon the aspiration for the NHS to be 'the first mainstream health service in the world to offer genomic medicine as part of routine care' (www.genomicsengland.co.uk). It aims to ensure a legacy for the NHS in the form of a genomics diagnostics service, which has the potential to improve the precision and quality of care delivered to patients. Internationally, the literature make a compelling case that the current nature and extent of genetics education for health professionals are rate-limiting factors in the integration of genetics into mainstream healthcare. ${ }^{7,12,13,21,22}$ Therefore, the success of this and other projects aiming to incorporate genomics into medical practice will be dictated, to a large degree, by the education of the health service workforce.

The Health and Social Care Act 2012 brought about change to many of the structures within the NHS. Among these were organisations underpinning responsibility for postgraduate medical education, including genomics education, in the UK. In January 2014, the Joint Committee on Genomics in Medicine (JCGM), the Public Health Genomics Foundation and the NHS National Genetics and Genomics Education Centre (in June 2014 the National Genetics and Genomics Education Centre joined Health Education England to form the Genomics Education Programme) formed a working group to raise awareness and champion the integration of genomics into clinical practice across a wide range of medical specialties. Investigation of professional medical education in the area of genomics identified a complex landscape, currently in development, with multiple stakeholders, who are often unaware of the view and work being undertaken at different points on the landscape. As part of the work of this group, this commentary seeks to define the current landscape of genomics education for specialists within medicine in England, providing readers with an overview of the complexity and an outline of the organisations involved.

\section{Structure of postgraduate medical training in the UK}

In July 2014, there were approximately 230,000 licensed doctors on the list of registered medical practitioners. These medical professionals comprise a highly heterogeneous population practising within 61 specialties. ${ }^{23}$ Specialty professional medical training for these doctors in the UK is coordinated through a three-constituent network: the General Medical Council (GMC), the royal colleges and local education and training boards (LETBs; Fig 1).

The GMC functions to protect, promote and maintain the health and safety of the UK public by ensuring proper standards in the practice of medicine. One of the ways in which it achieves this is through its regulation of medical education and training. The GMC has assumed overall responsibility for postgraduate medical training since absorbing the Postgraduate Medical Education Training Board (PMETB) in 2010. As an organisation, it sets the standards for the postgraduate training of UK medical professionals and then monitors the attainments of these standards through its quality-assurance work. Additionally, the GMC has a central regulatory role both in undergraduate education and in continuing professional development. $^{24}$

The royal colleges and faculties (grouped together under the aegis of the Academy of Medical Royal Colleges) set and maintain their specialty curricula and assessments for postgraduate training in the UK within the standards set by the GMC. Each college has unique arrangements for this. Additionally, they have a role in maintaining records of individual practitioner's continuing professional development for the purposes of revalidation and relicensing. ${ }^{24}$ 
As part of the reconfiguration of the NHS structures, set out in Liberating the NHS: Developing the healthcare workforce, from design to delivery, the delivery of postgraduate medical education within the NHS in England is now the responsibility of Health Education England (HEE), which became operational in spring 2013. ${ }^{24,25}$ Similar organisations exist in the other three constituent parts of the UK. The work of HEE is delivered at a regional level in England by LETBs, who oversee and manage training posts at NHS trusts and GP practices within their area, and are headed by a postgraduate dean. ${ }^{24}$ It is within HEE that a framework for the delivery of genomics education is being developed under the auspice of the Genomics Education Programme (for a wide range of the healthcare workforce, not just medical professionals), for implementation by other bodies within the medical training sphere.

\section{Framework for the delivery of genomics education to medical professionals}

The framework for the delivery of genomics education and training for the healthcare workforce in England builds on early work undertaken by the NHS Genetics Education and Development Centre, which was set up as part of NHS initiatives arising from the 2003 government white paper on genetics. ${ }^{17}$ The main organisations that make up this framework and are contributing to the direction of genomics education for healthcare professionals are HEE's Genomics Education Programme, the National Genetics and Genomics Education Centre (now part of the National School of Healthcare Science) and the JCGM.

As part of the government strategy on genomics, HEE was specifically challenged to ensure that 'the workforce, at every level, understands the opportunity genomics brings, that they possess the knowledge, skills and competence to deliver this care effectively and compassionately'. The first steps were formalised by the government (April 2013-March 2015) through a mandate to 'deliver a training strategy for genomics'. In response, in November 2013, HEE launched the Genomics Advisory Board (now stood down), with a remit to set the strategic direction and provide oversight for the agreed work programme and its implementation. The programme had a particular focus on the education and training implications of the 100,000 Genomes Project and would include the entire healthcare workforce in its scope (including, for example, laboratory and bioinformatics elements), as well as both specialist and more generalist elements. The Genomics Advisory Board had wide membership, including representatives from main partner organisations (Genomics England, Department of Health, the Royal College of Physicians (RCP), the Royal College of Pathologists, education providers, patient and public groups, research groups, charities and the commercial sector).

During its period of operation, the Genomics Advisory Group set up task and finish groups on clinical bioinformatics, educational resources, implementation of education, developing research and academic capacity, and consideration of patient and public viewpoints. Practical developments included the development of online introductory materials on genomics and bioinformatics (www.genomicseducation.hee.nhs.uk) and the launch of a new master's degree in genomic medicine aimed at
Box 1. Learning outcomes in genetics and genomics for specialty trainees in non-genetic specialties.

Describe the structure, function, and transmission of the human genome, and how its expression is regulated through genetic and epigenetic mechanisms (as the basis for understanding human diseases).

Be able to describe and differentiate the types and relative frequencies of variations in the human genome, how they arise, and how they contribute to health and disease through their effects on gene expression.

Know the genetic or genomic basis for disorders encountered in the specialty, and whether they follow a specific mode of inheritance.

Recognise, in conjunction with family history or as an isolated presentation, the subset of patients whose condition has a genetic basis (eg as a single gene condition), and institute appropriate genetic management for them and their family.

Order tests using genetic technologies appropriately, interpret the results and apply them in the context of clinical management.

Be able to communicate genetic and genomic concepts, probability information, and test results, to facilitate engagement and choice for the patient and their family in managing their condition.

Adapted from National Genetics and Genomics Education Centre. ${ }^{26}$

the range of health professionals involved in multidisciplinary teams. Delivery of the master's programme (and individual modules) is provided by a network of nine higher education institutes, geographically spread across the country, which started in September 2015. It is envisaged that this course, which has modules in rare diseases, cancers, infectious diseases and pharmacogenomics, will be of interest to doctors from a wide range of specialties and others from across the healthcare workforce.

The Genomics Education Programme aims to drive and coordinate genetics and genomics education for all UK health professionals, including those working in specialist genetic services (www.genomicseducation.hee.nhs.uk). It encompasses work previously developed by the National Genetics and Genomics Education Centre and includes formal statements of learning outcomes for genetics and genomics for specialty trainees in non-genetic specialties (Box 1). ${ }^{26}$ It also includes a wide range of online and printed resources (eg e-learning modules, point-of-care information and tools, curricula, teaching aids) to support the education of health professionals in genetics and genomics.

The JCGM is an organisation formed with representation from the RCP, the Royal College of Pathologists and the British Society for Genetic Medicine. It advocates for and supports high standards of practice in the clinical and laboratory applications of genetics to medicine. Its activities include consultation on policy and service issues, workforce planning and partnering in the development of professional training and education. 
Together, the work emanating from these three groups will inform and influence the strategic foundation upon which genomic medicine training for medical professionals in the UK is built. Successful implementation, however, will require the active engagement and participation of all organisations involved in the regulation and delivery of professional medical education and training (Fig 1).

\section{External bodies with a role in education and training medical professionals}

Independent to these core structures, there are two broad sets of organisations that have a potential role in genomic education: those with direct roles in postgraduate professional medical education, for which genomics might form a part of the educational content delivered, and those that have an important advisory or direct educational role within the field of genomic medicine, but are not directly responsible for the strategic direction and delivery of genomics education for medical professionals.

The former set of organisations includes the numerous bodies involved with aspects of professional medical education, training and continuing professional development in the UK that, through the course of their work, will be increasingly addressing issues of structuring and delivering education in genomic medicine. These include the subsidiary groups of organisations outlined in Fig 1 (eg Joint Royal Colleges of Physicians Training Board, Joint Committee on Surgical Training), higher education providers (eg St George's Hospital University of London has recently launched a massive openaccess online course, 'A new era: the future of genetics in medicine', which aims to give healthcare professionals a basic grounding in genomic medicine (www.futurelearn.com/ courses/the-genomics-era)); specialty associations (eg Royal Society of Medicine, British Society of Gastroenterology), research bodies (eg Medical Research Council, Wellcome Trust), charities (eg Cancer Research UK), and trade associations (eg Association of the British Pharmaceutical Industry). Several of these organisations already have internal initiatives or groups in place considering the future role of genomics in their work. However, exactly what projects are ongoing, what work has already been accomplished in this area and how much of the work of these groups is duplicated remain unclear.

One example of such a group is the medical genetics section of the Royal Society of Medicine, which seeks to facilitate cross-specialty education, integration and progress in the field of genetic medicine and to provide insight into and promote recent advances in genetic medicine as a cross-cutting discipline. Section meetings and educational events (eg 'The genetics of movement disorders' in January 2015) are run throughout the year to further these aims, and the membership currently comprises clinical geneticists, neurologists and rheumatologists. The value of such initiatives and the many professional conferences in which genomic advances are likely to be presented as cutting-edge clinical practice should not be underestimated in the promotion of genomic awareness and competence within medical specialties.

The second set of organisations includes independent or advisory bodies such as the Public Health Genomics
Foundation, which focuses on the translation of genomic advances into interventions and systems that can improve health, and the UK Rare Diseases Strategy and Stakeholder Forum, which commits to improve education and awareness of rare diseases across the healthcare professions. There are also centres that specialise in public and professional engagement and education in biomedical sciences, such as the Centre for Life in Newcastle (provides public engagement and educational events open to all, including medical professionals, such as the 'Genes in a bottle' workshop) and the Wellcome Trust Genome Campus in Cambridge, which provides science education from informal days and workshops to more formal advanced science courses such as 'Genomics and clinical microbiology' (delivered in January 2015).

Finally, there are a number of commercially developed websites, including sites such as Doctors.net, GPnotebook and BMJ Learning. These sites might have little separately categorised genomics education at present, but can be anticipated to incorporate it into their education modules on specific types of medical disorders.

\section{Discussion}

The discourse around workforce education in genomic medicine has gained momentum across a period of corporate and structural reconfiguration within the NHS, which, alongside significant budgetary pressures, has challenged progress. Additionally, there are specific issues relating to the building of a genomics knowledge base within the medical workforce. Firstly, the call for greater genomics education is not occurring in a vacuum, and there is great pressure on the curriculum for postgraduate medical training to address changing patterns of healthcare, as reflected in the Shape of Training Review (2013). ${ }^{27,28}$ This could be addressed by exploiting the cross-cutting nature of genomics, incorporating it into the learning opportunities provided by everyday clinical practice as a way to minimise the opportunity cost of this additional curriculum. ${ }^{20}$

Secondly, the work of curriculum development is occurring in the context of continuing, rapid progress in genomic medicine. ${ }^{20,22,27}$ The consistent and rapid evolution of the field needs to be accounted for when designing an educational strategy - the medical workforce will require a mechanism to keep up-to-date both in diagnostics and therapeutic strategies, ${ }^{3,12,29}$ including understanding of individual responses to medicines (pharmacogenomics) and the use of genomic tumour testing for precise molecular diagnosis of cancer and monitoring of treatment response. This is a challenge that is faced from undergraduate through to professional education and, although beyond the scope of this piece, the importance of medical undergraduate education in genomic medicine cannot be understated - they are the medical workforce of tomorrow, who will be required to understand and apply genomic medicine within their practice.

Thirdly, it has been argued that the lack of robust clinical evidence has impeded both education programmes and adoption of genomic advances by the wider medical community. ${ }^{2}$ Clinical genetics originally evolved to diagnose and manage patients and their families with rare inherited 
diseases - the low prevalence of such rare diseases prohibited the undertaking of large, prospective trials of diagnostics and therapeutics, the precepts of evidence-based medicine to which medical professionals adhere. Some have argued that, as new genomics technologies have emerged, the genomics community has accepted them as beneficial on the basis of little direct clinical evidence, with the rapid pace of discovery compounding the problem. ${ }^{2}$ Medical professionals are relentlessly practical: clinical utility and additional benefit to their practice will need to be demonstrated, before a favourable economic analysis is also demonstrated.

Until recently, most genomic advances were relevant to only a small subset of clinicians but the situation is changing rapidly. ${ }^{2,3,8,13}$ Clinicians do not need to become geneticists to utilise genomic information and practise personalised medicine any more than they need to be a radiologist to utilise complex imaging technology. ${ }^{2}$ However, as in this same analogy, the report of a genomic test result must be ratified by a specialist professional, who will usually be a clinical scientist, and should include guidance on appropriate interpretation for clinical application. Alongside this, to ensure the effective delivery of genomics within the healthcare service, the medical workforce requires a sound genomics knowledge base that addresses competencies within the specific areas of content required for their practice and accommodates the rapidity of change in the field. In the UK, an RCP working group comprising specialists in 15 different clinical specialties is currently developing introductory resources tailored for each individual specialty (www.phgfoundation.org/project/mainstream_medicine/). Several organisations have worked to define the core competencies required by clinicians both in the UK and across Europe. ${ }^{9,12}$ Furthermore, there have been examples of success in the education of mainstream medical professionals using online materials and e-learning, which could offer a cost-effective and time-efficient method that is favoured by the busy medical workforce. ${ }^{7,14}$

Recent precipitous change in the structure of postgraduate education has settled, but the landscape of genomics education in England, outlined here, as well as in Scotland, Wales and Northern Ireland, is complex and constantly developing. The large and growing number of organisations with an interest in the area of genomics could lead to unnecessary duplication of work and a lack of coherent educational resources available to clinicians. It is imperative that there is clear leadership and coordination in both the determination and delivery of a national genomics education strategy. In the new structure, the Genomics Education Programme of HEE is well placed to adopt this coordination and leadership role within England. Clear and timely communication of this strategy and its developments, as well as clarity about which groups will assume responsibility for the various initiatives, will be crucial to the success of this endeavour.

A national education strategy (for the medical workforce) to be delivered within the NHS will require designated and sustained investment. Clinicians will require guidance beyond competencies and curricula so that genomic medicine is built into clear guidelines and definitions of their responsibilities in practice. ${ }^{7}$ Furthermore, there will need to be collaboration with those responsible for the education of the next generation of medical professionals so that the future is assured.

\section{Conclusion}

Genomic science, incorporated into the national healthcare landscape, offers the potential of improved patient care and disease prevention that can be fully exploited only by an educated health professional workforce. Cooperation and coordination will be required both between the multiple strategic and delivery players outlined in this review, as well as with the biomedical research sector, to effectively nurture such a workforce. Furthermore, any national educational strategy will need both persistence and substantial sustained resource commitment. Education will be the key if the full measure of health benefits from genomic science is to be achieved. Future work might involve contacting these groups and organisations to gather information on genomics initiatives that they have set up themselves, and to seek the involvement of those who are yet to develop policies in this area.

\section{Conflicts of interest}

The authors declare no conflicts of interests.

\section{Acknowledgements}

We gratefully acknowledge input of members of the Steering Group on Genomics in Mainstream Medicine: Prof John Wass, Dr Trevor Cole, Prof Ian Cree, Prof Peter Farndon, Dr Bronwyn Kerr and Dr Sarah Smithson. The work of the Centre for Personalised Medicine at the University of Oxford is supported by funding from the Dr Stanley Ho Medical Development Foundation. HB and DS are supported by the Public Health Genomics Foundation.

\section{References}

1 Boycott KM, Vanstone MR, Bulman DE, MacKenzie AE. Raredisease genetics in the era of next-generation sequencing: discovery to translation. Nat Rev Genet 2013;14:681-91.

2 Feero WG, Green ED. Genomics education for health care professionals in the 21st century. JAMA 2011;306:989-90.

3 Academy of Medical Sciences. Realising the potential of stratified medicine. London: Academy of Medical Sciences, 2013.

4 Manolio TA. Genomewide association studies and assessment of the risk of disease. N Engl J Med 2010;363:166-76.

5 McDermott U, Downing JR, Stratton MR. Genomics and the continuum of cancer care. N Engl J Med 2011;364:340-50.

6 Relling MV, Evans WE. Pharmacogenomics in the clinic. Nature 2015;526:343-50.

7 Houwink EJ, van Teeffelen SR, Muijtjens AM et al. Sustained effects of online genetics education: a randomized controlled trial on oncogenetics. Eur J Hum Genet 2014;22:310-16.

8 Burton H. Genetics and mainstream medicine. Service development and integration. Cambridge: Public Health Genomics Foundation, 2011.

9 Skirton H, Lewis C, Kent A, Coviello DA, Members of Eurogentest Unit 6 and ESHG Education Committee. Genetic education and the challenge of genomic medicine: development of core competences to support preparation of health professionals in Europe. Eur J Hum Genet 2010;18:972-7.

10 Rahman N. Mainstreaming genetic testing of cancer predisposition genes. Clin Med 2014;14:436-9.

11 O'Sullivan J, Mullaney BG, Bhaskar SS et al. A paradigm shift in the delivery of services for diagnosis of inherited retinal disease. J Med Genet 2012;49:322-6.

12 Farndon PA, Bennett C. Genetics education for health professionals: strategies and outcomes from a national initiative in the United Kingdom. J Genet Counsel 2008;17:161-9. 
13 Challen K, Harris HJ, Julian-Reynier C et al. Genetic education and nongenetic health professionals: educational providers and curricula in Europe. Genet Med 2005;7:302-10.

14 Burke S, Stone A, Bedward J, Thomas H, Farndon P. A "neglected part of the curriculum" or "of limited use"? Views on genetics training by nongenetics medical trainees and implications for delivery. Genet Med 2006;8:109-15.

15 Feero WG, Manolio TA, Khoury MJ. Translational research is a key to nongeneticist physicians' genomics education. Genet Med 2014;16:871-3.

16 Haga SB, Burke W, Ginsburg GS, Mills R, Agans R. Primary care physicians' knowledge of and experience with pharmacogenetic testing. Clin Genet 2012;82:388-94.

17 Department of Health. Our inheritance, our future. Realising the potential of genetics in the NHS. London: Department of Health, 2003.

18 House of Lords Science and Technology Committee. Genomic medicine. London: House of Lords, 2009.

19 Burton H, Wright CF, Zimmern R. A new strategic phase for genomic medicine in UK health services. Genome Med 2009;1:93.

20 Burton H, Cole T, Farndon P. Genomics in medicine. Delivering genomics through clinical practice. Cambridge: Public Health Genomics Foundation, 2012.

21 Guttmacher AE, Porteous ME, McInerney JD. Educating healthcare professionals about genetics and genomics. Nat Rev Genet 2007;8:151-7.
22 McInerney JD. Genetics education for health professionals: a context. J Genet Counsel 2008;17:145-51.

23 Council General Medical. Specialties, sub-specialties and progression through training: the international perspective. London: General Medical Council, 2011.

24 Black D. A brief guide to who does what in postgraduate medical education. Commentary 2014;3:18-19.

25 Ovseiko PV, Jenkinson C, Buchan AM. Medical education leaders' perceptions of postgraduate medical education reform. Lancet 2014;384:306-7.

26 National Genetics and Genomics Education Centre. Learning outcomes in genetics and genomics for specialty trainees in non-genetic specialties. London: National Genetics and Genomics Education Centre, 2014.

27 Greenaway D. Securing the future of excellent patient care. Shape of Training, 2013. London: General Medical Council, 2013.

28 Council General Medical. The state of medical education and practice in the UK. London: General Medical Council, 2011.

29 Passamani E. Educational challenges in implementing genomic medicine. Clin Pharmacol Therapeut 2013;94:192-5.

Address for correspondence: Dr I Slade, The Ethox Centre, Nuffield Department of Population Health, University of Oxford, Old Road Campus, Oxford OX3 7LF, UK.

Email: ingrid@well.ox.ac.uk
'This landmark report lays out in the starkest terms yet the devastating impact air pollution is having on our health, our economy and our society as a whole.'

\section{Every breath we take The lifelong impact of air pollution}

This major report plainly sets out the dangerous impact that air pollution has on our nation's health. Compiled by experts in medicine and environmental sciences, the report discusses the current evidence and draws up recommendations for action.

ISBN 978-1-86016-567-2 £15 including $\mathrm{p}+\mathrm{p}$ or free to download

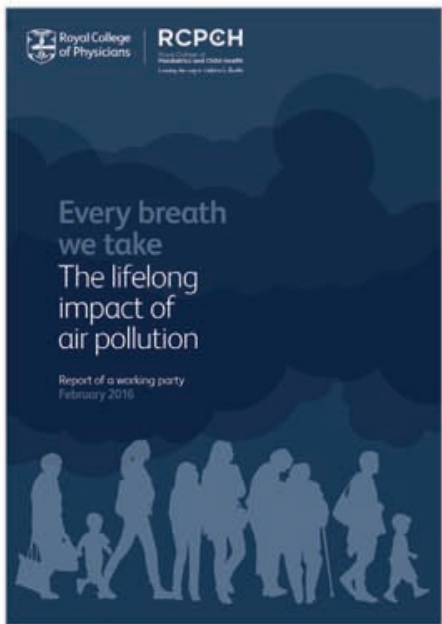

Order a copy: shop.rcplondon.ac.uk 